\title{
Utility of whole-exome sequencing in pediatric and medical diagnosis
}

Mohd Fareed ${ }^{1,2 *}$, Varun Sharma ${ }^{3}$, Hemender Singh $^{4}$

${ }^{1}$ Academy of Scientific \& Innovative Research (AcSIR), Ghaziabad, Uttar Pradesh, 201002, India

${ }^{2}$ PK-PD Formulation \& Toxicology Division, CSIR Indian Institute of Integrative Medicine, Canal Road, Jammu,180001, India

${ }^{3}$ Ancient DNA Laboratory, Birbal Sahni Institute of Palaeosciences, Lucknow, Uttar Pradesh, 226007, India

${ }^{4}$ School of Biotechnology, Shri Mata Vaishno Devi University, Katra Jammu \& Kashmir, 182320, India

*Corresponding author (M. Fareed) Tel.: +91-9622025886

Email address: mohdfareedk@gmail.com; m.fareed@iiim.res.in

\section{Impact}

- WES is a cost-effective technology compared to WGS in the diagnosis of pediatric-onset genetic diseases.

- WES is effectively uncovering genome-based drug targets for precision and personalized medicine.

- WES appropriately explicates heterogeneous disorders and offers rich evidence-based guidelines that assist families in genetic counseling. 


\begin{abstract}
Genetic disorders are preeminent determinants of infant mortality. The inherited pediatric-onset genetic disorders have consequential stress on child growth and development: several congenital, complex and rare disorders with indistinguishable clinical symptoms where diagnosis always remains a challenging task. Traditional diagnosis methods include biochemical tests followed by chromosomal microarray and sequencing of a single gene or panel of genes. These methods had several limitations, but with the advent of whole-exome sequencing (WES), genetic testing has become cost-effective and transformative. Exome sequencing has been known for its effectiveness, which appropriately elucidates and distinguishes the heterogeneous disorders to avoid misdiagnosis and decode the underlying genetic alterations. WES has led to discovering genes and genomic variants in a broad spectrum of diseases, including autism, epilepsy, congenital heart diseases, neurodevelopmental diseases, cancer, nephrotic disorders, neural tube defects and fetal structural anomalies. WES is significant in producing immense genomic biomarkers that can be made as appropriate pharmacogenomic targets for drug therapy. In this article, we analyze the recent exploration of WES technology to revolutionize not only the process of genetic variation and disease detection but also the convention of preventative and targeted drug discovery.
\end{abstract}




\section{INTRODUCTION}

Recent estimates suggest that 8 million newborns each year have a severe congenital disability of genetic or a condition with somewhat genetic influence. Further, low and middle-income countries contribute more than $90 \%$ of the global infant mortality due to congenital disabilities [1]. The most common genetic ailments include single-gene disorders, copy number variants (CNVs), structural and functional abnormalities, which mostly originate during the first trimester of pregnancy. Approximately 2-3\% of fetal anomalies during pregnancies are responsible for about $20 \%$ of the perinatal deaths [2]. The infant and child mortality due to congenital disabilities influence disproportionately among different populations worldwide [1]. Annually, congenital disabilities causing the death of 1 in 3 before the age of 5 and those who survive may suffer lifelong mental and physical health $[1,3]$.

Several congenital, complex and rare disorders with indistinguishable clinical manifestations remain a question mark for the diagnostic odyssey. The heterogenicity of complex and rare diseases asserts a distractive burden on clinical or medical interpretation. Rare diseases affect human healthcare in physical, sensory, neurological, respiratory, primary immunodeficiency (PID) and intellectual disability (ID) with a varied extent of severity [4,5]. Establishing early genetic testing intends clinicians to intervene in proper diagnosis and optimal patient management, thus building confident decisions for the child's development [6].

Genome scanning has now been an exigent need to reveal the genetic determinants of the disease. Sequencing and scanning genomic variants for common and rare diseases has become feasible with subsequent human genome mapping and massively parallel next-generation sequencing (NGS) [7]. Whole-exome sequencing (WES) exclusively targets the protein-coding regions of the genome, providing a high diagnostic yield compared to conventional genetic testing methods [8]. WES covers about 30 million bases in the human genome that lead to the translation into a functional protein, resulting in direct phenotypic outcomes [9]. Compared to the whole genome sequencing (WGS), WES (covers 1-2\% of the genome) is cost-effective and data generated is easy to analyze, whereas the cost ultimately becomes higher in WGS, due to the generation of a large amount of data that eventually need high-performance computers and bioinformaticians [10]. It is interesting to know that the first successful use of exome sequencing in diagnosing a rare Mendelian disease; Miller syndrome [11]. Since then, WES has been used extensively in analyzing several genetic anomalies and has led to the identification of many novel genes and variants associated with many disorders [12]. 
Traditional diagnostic methods (fluorescent in-situ hybridization, karyotyping, chromosomal microarray, etc.) had many limitations due to their lower resolution. But with the introduction of NGS, the resolution increases up to single base pair with a high diagnostic yield for individuals suffering from genetic disorders [13]. WES has led to discovering genes and genomic variants in a broad spectrum of diseases namely; autism, malformations of the brain, epilepsy, congenital heart diseases, neurodevelopmental diseases, neural tube defects and fetal structural anomalies [14-20]. Figure 1 presents an overview of the genetic diseases and complete workflow of diagnosis using exome sequencing.

This review target the use of WES in the diagnostic expedition of several pediatric-onset genetic diseases. Here, we tried to highlight some of the studies where WES significantly diagnosed specific illness and led to identifying novel genomic signatures of the disease.

\section{AUTOSOMAL RECESSIVE DISORDERS}

Autosomal recessive disorders (ARDs) are generally found in the offspring of consanguineous couples [21]. Consanguineous marriage is the marriage between a second cousin and even close relation in a family. Studies have reported the structural (physical) and mental (cognitive) health issues among children from consanguineous families [22,23]. Increased child mortality (prenatal and postnatal) rates have been observed among inbred Northern Indian families [24].

Most of the time, the ARDs are being misdiagnosed, where some remain undiagnosed. But with the advent of WES technology, it becomes easier to diagnose the problem at the earliest. Various studies have been carried out to identify and diagnose recessively inherited diseases. Long QT syndrome (LQTS) is a heart rhythm disorder that can potentially cause irregular heartbeat, which consequently results in fainting, seizures, or sudden death. Clinicians and medical practitioners very often misdiagnosed LQTS with a seizure disorder, such as epilepsy. Altmann et al., in 2015, used WES and was successfully able to identify the novel frameshift gene mutation in the TRDN gene in LQTS among $\leq 10$ years of aged children and in older patients [25]. Autosomal recessive spastic ataxia of Charlevoix-Saguenay (ARSACS) is a neurodegenerative disorder characterized by earlyonset cerebellar ataxia. Some recent studies using WES have identified novel homozygous pathogenic mutations in the $S A C S$ gene [26,27].

A study carried out by Sawyer et al. has identified 28 families with pediatric-onset ataxia, of which WES was done in 13 families and a success rate of 46\% was achieved. Where in 11 families, they succeeded in identifying mutations in the genes that were reported to be associated with known neurological syndromes and in two of the families, they identified novel disease genes. This study suggests that exome sequencing is an effective approach for pediatric patients with ataxia, where a 
particular single gene is not suspected to be causative [28]. It is well known that ARDs risk increases in the offspring of consanguineous parents. In a study carried out by Makrythanasis et al., where they recruited 50 consanguineous families with no definitive molecular diagnosis. Exome sequencing and comparative genome hybridization (CGH) was performed and it was observed that 18 families were linked to a specific clinical phenotype. Interestingly, pathogenic mutations were identified through WES among 17 families and one family was diagnosed with CGH. This study showed the utility of WES compared to other techniques a better and useful tool in the diagnosis of ARDs [29].

In another study on malignant infantile osteoporosis (MIOP), a rare congenital ARD, categorized by increased bone density due to impaired bone resorption by osteoclasts. WES was carried out among six patients and was successfully able to identify mutations in four genes: $S N X 10$, TNFRSF11A, TCIRG1 and CLCN7. Mutations in five children were found to behave homozygous, whereas, in the additional one patient, the compound heterozygous mutations in the TCIRG1 gene were identified [30]. Another ARD, the Caroli disease which is complex and rare and is characterized by cystic dilatation of intrahepatic bile ducts. It mostly leads to jaundice, cirrhosis, dilatation of renal tubules, as well as renal impairment in children and is also found to be associated with multicystic or polycystic kidney disease. A study on this disease by Hao et al. in 2014 revealed the importance of WES in diagnosing the condition. They were able to identify the genetic defect in a Chinese twin family with a novel compound heterozygous mutations in PKHD1. A missense mutation c.2507 T.C, with a substitution of valine to alanine at codon 836 (c.2507T $>$ C, p.Val836Ala), and a nonsense mutation c.2341C.T, causing arginine to stop codon at codon number 781 (c.2341C>T, p.Arg781*) were predicted to drive the pathogenicity of the disease [31]. 


\section{X-LINKED DISORDERS}

$\mathrm{X}$-linked disorders are found to be having a low prevalence in females, whereas males are severely affected by a degree of penetrance. To date, more than hundreds of X-linked disorders have been well established [32]. We, at this moment, reviewed a few studies on X-linked disorders in context to the use of WES in their diagnosis.

A study on autism spectrum disorder (ASD), using WES in 19 case parent trios recruited from Saudi families, revealed 47 rare variants in 17 trios; 38 were novel. The majority of these variants were X-linked or autosomal recessive. They exposed the variants from 15 ASD genes, including 5 genes (GLT8D1, HTATSF1, OR6C65, ITIH6 and DPX26B) that indeed have not been described in any of the human ailments [33]. Another study on neonatal encephalopathy (generally occurs 17/1000 live births), characterized by a decrease in the level of consciousness. Out of 14 patients, five were confirmed through WES. They identified autosomal dominant variants in SCN2A (p.Met1545Val), GNAO1 (p.Gly40Arg) and KCNQ2 (p.Asp212Tyr) genes associated with epileptic encephalopathy. Compound heterozygous variants in LIAS (p.Ala253Pro and p.His236Gln) gene related to the deficiency of lipoic acid synthetase and X linked variant associated encephalopathy in $C U L 4 B$ (p.Asn211Ser) gene [34].

A study of 78 patients, based on neurodevelopmental disorders, using the WES platform revealed a diagnostic rate of $41 \%$ (i.e., 32 patients) of the cohort. The observations were autosomal dominant $(n=19)$, autosomal recessive $(n=11)$, X-linked $(n=01)$ and one case with both autosomal dominant and recessive disorder. The WES uncovered the harboring pathogenic variants among the patients showing neuroimaging and neurobehavioral abnormalities [35]. Yang et al., 2014 performed clinical WES to decipher the Mendelian disease patterns in 527 patients. The findings were $280(53.1 \%)$ autosomal dominant, 181 (34.3\%) autosomal recessive (including 5 with uniparental disomy), 65 (12.3\%) X-linked, and 1 (0.2\%) mitochondrial. This study showed that the potentiality of WES might offer an advantage over other traditional molecular diagnostic approaches in terms of better yield and proper diagnosis [36].

The neurodevelopmental delays accompanied by unexplained dyspnea is exceptionally fatal. A study on such patients using Trio-WES has reported eight genes namely G6PC, MMACHC, G6PT, OTC, ETFDH, NDUFAF5, MAGEL2 and SLC22A5 which were found to be responsible for the clinical symptoms. In this study, 6 novel variants have been reported, indicating WES effectiveness in exploring the hidden hallmarks of neurodevelopmental disorders [37]. 


\section{MITOCHONDRIAL DISORDERS}

Mitochondrial dysfunction is a primary cause of metabolic disorders in adults and children. Mitochondrial DNA (mtDNA) mutations may cause a wide range of diseases, which explicate approximately $10 \%-30 \%$ by such mtDNA changes while others modulated by imperfections in nuclear-encoded mitochondrial proteins [38]. The first reported evidence of the mitochondrial disorder was found in 1959, where a woman aged 30 years had clinical symptoms that were developed when she was 7 years old. The symptoms mostly include increased calorie intake, high rate of perspiration, increased fluid intake but with no condition of polyuria and muscular weakness and she was diagnosed with a mitochondrial disease later named Luft disease [39].

It is well known that the offspring predominantly constitutes maternal mtDNA. Any mutation in the mitochondrial genome or nuclear genome may give rise to anomalies in the offspring. Mitochondrial disorders may arise because of any dysfunction in the respiratory chain [40]. Generally, mitochondrial disorders may occur at any age but seem critical to diagnose at an early life phase [41]. These disorders may affect either a single organ or multiple organs. Some of the commonly diagnosed mitochondrial disorders are Leigh syndrome (LS), chronic progressive external ophthalmoplegia (CPEO), myoclonic epilepsy with ragged-red fibers (MERRF), KearnsSayre syndrome (KSS), neurogenic weakness with ataxia and retinitis pigmentosa (NARP), or mitochondrial encephalomyopathy with lactic acidosis and stroke-like episodes (MELAS) [40]. The prevalence of Childhood-onset $(\leq 16$ years of age) mitochondrial oxidative phosphorylation (OXPHOS) disorders ranges from 5 to 15 per 100,000 individuals [42].

The first diagnostic criterion for mitochondrial disorders was proposed by Walker et al., 1996 [43], followed by some modifications made in a few decades [44]. It has been observed that most of the mitochondrial disorders in children are nuclear in origin [42]. It is estimated that over 1000 nuclear genes encode mitochondrial proteins. If we need to name one useful tool for the genetic diagnosis of mitochondrial disease, the first name comes out to be WES technology [45]. WES has helped us in identifying novel pathogenic variants of mitochondrial proteins with known and unknown functions. Currently, the WES approach is being widely used and overcoming wholegenome sequencing (WGS) in some aspects [46].

Discussing the importance of WES in mitochondrial disorders can be well understood by expounding individual studies. One of the studies carried out by Pronicka et al. in 2016 performed molecular diagnosis by using WES on 113 patients in Poland. The WES was performed, and it was found that $50.5 \%$ of the causative mutations account for novel variants. In 47 subjects, variations in 31 mitochondrial disorders related genes (ACAD9, ADCK3, AIFM1, CLPB, COX10, DLD, EARS2, FBXL4, MTATP6, MTFMT, MTND1, MTND3, MTND5, NAXE, NDUFS6, NDUFS7, NDUFV1, 
OPA1, PARS2, PC, PDHA1, POLG, RARS2, RRM2B, SCO2, SERAC1, SLC19A3, SLC25A12, TAZ, TMEM126B, VARS2) were identified [47]. Using WES, a study unraveled the infrequent mitochondrial disorders among three patients. The clinical symptoms were complicated to decide the disease etiology. WES was performed and different mutations were observed in each case viz., patient 1 (p.Glu415Gly and p.Arg484Trp variants in MTO1 gene), patient 2 (p.Gln111ThrfsTer5 and RNA mis-splicing in TSFM gene) and patient 3 (p.Met151Thr and p.Met246Lys variants in AARS2 gene) [45]. Gotz et al. reported tRNA synthetase mutation in an infant suffered from cardiomyopathy. The child died at the age of 10 months. The WES was done to identify the molecular basis of the pathology, and it was reported that there was a homozygous missense mutation (c.1774C $>$ T, p.Arg592Trp) in the AARS2 gene that encodes the mitochondrial alanyltRNA synthetase. Similarly, two siblings from another family who also died perinatally because of hypertrophic cardiomyopathy were found to be encountered with the same mutation, and it was compound heterozygous with another missense mutation. The study well exposed the significance of WES in identifying the mutations in single-gene disorders [48]. In another study, where 53 patients were confirmed with biochemical evidence showing multiple respiratory chain complex defects with no mtDNA mutation reported. Presumed causal variants were identified in 28 patients, where possible causal variants in 4 individuals. About 18 different genes were involved in these patients including mutations in RMND1, AARS2, and MTO1 and mutations in 4 likely mitochondrial disorder genes namely VARS2, PTCD1, GARS and FLAD1 [49]. It is evident from the above studies that WES can even identify nuclear gene variants that may be associated with mitochondrial diseases. Similarly, numerous novel pathogenic variants related to mitochondrial genetic defects are now being deciphered using WES.

\section{CONGENITAL DISORDERS}

Congenital anomalies or congenital malformations often-inherited medical conditions that develop prenatally and occurs at or before birth. Genetic disorders or congenital disabilities are considered as the primary cause of death under the age of five [50]. These disorders may affect the structural or functional aspects of the body that may include metabolism and intellect. Approximately more than 7000 known congenital disabilities have a wide range of severity from mild to lethal abnormalities [51]. Here, We review some of the recent efforts made by WES to decode the etiology of pediatric-onset congenital disorders.

Congenital disorders of glycosylation (CDGs) are a group of inherited diseases that are caused by defects in the synthesis and processing of the asparagine (ASN)-linked oligosaccharides of glycoproteins. Congenital disorders of glycosylation type I (CDG-I) are also categorized as a group 
of recessive neurometabolic diseases [52]. In a study, the WES variants were prioritized based on the list of 76 potential CDG-I candidate genes, which had led to the identification of one known and two novel CDG-I gene defects. The first one was X-linked CDG-I due to a de novo mutation in $A L G 13$, the compound heterozygous mutations in DPAGT1, and mutation in PGM1 in two cases. The pathogenicity of the mutations was confirmed by showing the deficient activity of the corresponding enzymes in patient fibroblasts [53]. Phosphomannomutase 2 (PMM2-CDG) deficiency is the most frequent N-linked glycosylation disorder and subtypes among all CDG conditions. Clinical symptoms may involve abnormal coagulation, hepatopathy, intellectual disability (ID), cerebellar ataxia, and peripheral neuropathy. WES revealed the compound heterozygous (c.241-242 del) as a novel and a previously reported (c.395 T > C) variant in the PMM2 gene of Hani ethnic siblings [54].

Congenital anomalies and intellectual disability (CA/ID) is a major diagnostic challenge in medical genetics. Approximately 50\% of patients still have no molecular diagnosis after a long and stressful diagnosis. The reanalysis using the WES approach has now become a direct substitute for the families suffering from heterogenetic rare CA/ID, who remain undiagnosed by traditional diagnostic strategies [55]. A recent study with 62 families with congenital anomalies of the kidney and urinary tract (CAKUT) was recruited from Texas Children's Hospital, Houston Texas, USA. They performed WES and approximately $5 \%$ of families reported were having pathogenic single nucleotide variants (SNVs) in $H N F 1 B, P A X 2$ and $E Y A 1$. Additionally, four causative copy number variants (CNVs) were also identified. One deleterious de novo SNV in FOXP1 was also recognized among all the families with the disease [56].

Maturity-onset diabetes of the young (MODY) and congenital hyperinsulinism (CHI) are the two most prevalent monogenic disorders of glucose-regulated insulin secretion in childhood. To date, there are 13 causative genes familiar for MODY and ten causative genes identified for CHI. In a recent investigation, ten subjects with MODY and 5 with $\mathrm{CHI}$ with known mutations underwent WES. Four of five MODY mutations were identified including a large deletion in HNF1B. Although targeted, one mutation (in INS) had insufficient coverage for detection. All 11 mutations (6 MODY, 5 CHI) were identified using Nextera Rapid Capture Exomes (including the previously missed mutation). On reconciliation, all mutations identified with previous data, and no further variants in MODY genes were detected [57]. The above-reviewed studies on congenital disorders provide an insight into the use of WES in search of genomic instabilities (SNVs, CNVs, indels, etc.) for a better understanding of the etiology of the disease. 


\section{NEUROLOGICAL DISORDERS}

The nervous system is a complex and challenging organ system to study, and the brain is the organ where many of the most pervasive disease processes arise, for which the cause remains elusive. These disorders are generally caused by genetic mutations that may impair the development of the nervous system, impaired neuronal function or neurodegeneration [58]. The diagnostic excellence using WES in such disorders have been summarized below:

In a retrospective cohort study $(\mathrm{n}=78$ patients) with various neurodevelopmental disabilities, the diagnostic rate of $41 \%$ achieved using WES. Based on the type of mutation and inheritance pattern, the patients were classified as autosomal dominant (AD, $n=19)$, autosomal recessive (AR, $\mathrm{n}=11)$, X-linked dominant $(\mathrm{n}=1)$, and 1 with both an AD and an AR disorder. These patients acquire a spectrum of neurodevelopmental disabilities, such as developmental delay (DD), intellectual disability (ID), cerebral palsy (CP), autism spectrum disorder (ASD), language and learning disorders. The WES delivered a relatively high diagnostic yield which further helps in precise diagnosis and management [35]. WES performed on 264 subjects with infantile spasms or Lennox-Gastaut syndrome (LGS) exposed the high frequency of de-novo variants in a set of genes presenting a strong correlation with epilepsy [59].

Alternating hemiplegia of childhood (AHC) is a rare neurological disorder that is characterized by early-onset events of hemiplegia, dystonia, various paroxysmal symptoms, and developmental impairment. In a study, patients with clinically characterized AHC, trio-WES of three subjects were done to identify the disease-associated gene and then further tested in the remaining patients and their healthy parents. ATP1A3 was found to be the disease-associated gene in AHC. The diseaseassociated de-novo missense and de-novo splice-site mutations were observed in the ATP1A3 gene [60]. Childhood apraxia of speech (CAS) another rare neurological disorder, a persistent pediatric motor speech disorder with associated deficits in sensorimotor, cognitive, language, learning processes. Using WES as a molecular diagnosis of the 32 patients and a total of five chromosomes (Chr3, Chr6, Chr7, Chr9 and Chr17), were found to be associated with the condition that included six genes (ATP13A4, CNTNAP1, CNTNAP2, FOXP1, KIAA0319 and SETX) strongly associated with CAS. It was observed that a total of $8(80 \%)$ out of 10 participants harbor variants in one or two of the six genes, with variants in ATP13A4, KIAA0319 and CNTNAP2 being the most prevalent [61]. Similarly, a study was carried out on the spectrum of pediatric epilepsy, where they recruited 9 families. Applying WES, they identified the mutations in genes (ASAH1, FOLR1, GRIN2A, $S C N 8 A$, SYNGAP1 and SYNJ1) associated with known neurological and epilepsy disorders. A novel and rare mutation were reported in the $K C N Q 2$ gene which was likely to be responsible for benign seizures segregating in the family [62]. 


\section{DISEASES WITH CLINICAL AND GENETIC HETEROGENEITY}

Rare mutations are responsible for contributing to the considerable severity of complex human diseases. Evolutionary forces contribute to vast genetic heterogeneity in human illness by introducing many novel variants in every generation [63]. Current sequencing technologies specifically WES, offer the possibility of identifying rare disease-causing variants and the genes that harbor them [64]. Many studies have preferred WES as a molecular diagnostic tool for diagnosing the genetic heterogeneity of the disease.

In a study on the Tunisian population, 13 patients from 6 unrelated consanguineous families showing horizontal gaze palsy with progressive scoliosis (HGPPS) were genetically examined. It was found that four distinct homozygous mutations in the $\mathrm{ROBO} 3$ gene were responsible. Two of those mutations novel homozygous, causing effective damage to the protein by in-silico analysis [65]. Another disease with genetic heterogeneity is cerebral palsy (CP) a common, clinically heterogeneous set of disorders that affects the movement and posture of an individual. It is estimated that genetic involvement in CP causation had been predicted to be $\sim 2 \%$. In the study whole-exome sequencing was performed for a cohort of 183 Caucasian cases with CP. Interestingly, 61 de novo protein-altering variants in case-parent trios, ten de novo mutations in three previously reported diseased genes (TUBA1A, SCN8A and KDM5C), and six novel candidate CP genes (AGAP1, JHDM1D, MAST1, NAA35, RFX2 and WIPI2) were identified and predicted to be potentially pathogenic for the condition [66].

Another disorder is Leigh syndrome (LS), the most common childhood mitochondrial metabolic disorder, characterized by clinical and neuroradiologic features. A study with 64 patients from 62 families was recruited after clinically diagnosed with LS. Mitochondrial genetic analysis followed by WES was executed on 61 patients. Pathogenic variants in mtDNA were identified in 18 families and nuclear DNA mutations in 22 families. Further analysis on 17 genes (MTATP6, MTND1, SLC19A3, ECHS1, PNPT1, IARS2, MTND3, MTND5, MTND6, NDUFS1, NDUFV1, NDUFAF6, SURF1, MTTK, NARS2, VPS13D, and NAXE) in 40 families were found to have genetic complexity. Two individuals had biotin-thiamine responsive basal ganglia disease, and another 3 cases were identified with defects in the newly recognized genes (VPS13D or NAXE) [67]. The study showed the clinical and genetic spectrum of LS, showed genetic heterogeneity.

A study from Japan, where 26 infants diagnosed with hypomyelinating leukodystrophy based on clinical features and brain MRI findings. To uncover their genetic etiology different diagnostic approaches were used viz; chromosomal analyses, targeted gene analyses, and an array comparative genomic hybridization (aCGH) assay. It was found that the diagnostic rate of chromosomal studies, 
targeted gene analyses, and aCGH was $31 \%$ (8 out of 26 cases), and one patient was clinically diagnosed with Cockayne syndrome. But with the use of WES, four causative genes (TUBB4A, POLR3B, MCOLN1 and KCNT1) of hypomyelination were identified in 6 individuals i.e. $35 \%$ (6 out of 17), and some of those identified genes were pathogenic for not only hypomyelination but also dysmyelination or delayed myelination [68].

\section{UNDIAGNOSED AND RARE DISEASES}

Mendelian disorders (MD) are generally categorized as rare disorders and affect many individuals and families. Due to a lack of resources in diagnosis, prognosis and treatment, the rare disorders are neglected in many ways and usually termed as orphan diseases. Recent estimates suggest that there are more than 8000 distinct rare disorders exist. SNVs, insertion and deletions accompany these disorders and CNVs cause many undiagnosed conditions that are generally termed monogenic or single-locus disorders.

With the application of WES and bioinformatic tools, it is now feasible to determine novel genes responsible for rare undiagnosed disorders [69]. A pediatric study based on 50 children with the developmental illness of unclear etiology and nonspecific neurological expressions. Using WES as a diagnostic tool, they were able to identify the known disease-causing gene mutation in 21 children. Whereas, in another 22 children, they got success in detecting probable candidate genes that were not currently known to be associated with the human disorder. The study showed the significance of WES in pediatric neurology [70].

The National Institutes of Health (NIH) Undiagnosed Diseases Program (UDP) in 2012 worked out on 1191 patients from medical records, of which 326 were accepted for the study based on the criteria of selection. Out of the approved subjects, $47 \%$ were children. The diagnosis was carried out based on single-nucleotide polymorphism (SNP) array and WES. Interestingly, they were able to discover two new disorders and the diagnosis of some patients with rare and ultra-rare disorders and some with common ailments. One of the undiagnosed conditions was identified as arterial calcification ACDC (calcium build-up in the arteries and joints of the hands and feet) which is caused due to the deficiency of CD73 resulted from mutations in the NT5E gene and the other undiagnosed disease was found to be familial distal myopathy with a single mutation in HINT3 gene [71].

A study from Israel, where 57 pediatric patients who were previously undiagnosed underwent WES for their diagnosis which resulted in the identification of causative gene in $49.1 \%$ subjects. Seventeen cases were having the autosomal dominant mutation, 9 were having autosomal recessive, 
and 2 with X-linked mutation. This study also provides evidence regarding the use of WES in the identification and diagnosis of rare and undiagnosed disorders [72].

\section{SOMATIC DISEASES}

WES has also led to the discovery of many novel variants associated with several somatic diseases. Neuroblastoma is a common childhood mortality disorder that affects $10-12 \%$ of young children. To determine the role of somatic mutation in such conditions, a study was performed with the implementation of WES in the diagnosis of the cases; several somatic mutations were discovered that were associated with the prognosis of the disease and the tumor size. This also led to the hypothesizing the role of such mutations as a biomarker for evaluating the effect of chemotherapy and disease prognosis [73]. The human brain is highly vulnerable to somatic mutations and the rate is very high during neurogenesis. Several somatic mutations occur during the time of neurogenesis and led to the development of neurodevelopmental diseases. With the advent of NGS specifically targeted (including WES), it becomes possible to uncover several novel somatic variants associated with the disease pathology. There is a long list of somatic mutations in several associated genes in neurodevelopmental diseases. Some of them are double-cortex syndrome (DCX, LIS1), neurofibromatosis type 1 (NF1), neurofibromatosis type 2 (NF2), Rett syndrome (MECP2), etc [74].

Furthermore, a susceptible and accurate exome-based cancer test has also been approved by the New York state department of health. The test is Exome Cancer Test v1.0 (EXaCT-1), and it also has a validated pipeline for the detection of somatic mutations. This also provides valid evidence regarding the importance of somatic mutations and exome sequencing as an essential diagnostic tool in precision cancer care [75].

\section{UNCOVERING NEW DRUG TARGETS FOR PRECISION AND PERSONALIZED THERAPY}

The evolution of clinical diagnostics into molecular diagnostics (i.e., using high throughput sequencing techniques) has opened the doors for precision medicine for rare and undiagnosed disorders. Earlier, medication for rare diseases was defined based on symptoms an individual harbors. Currently, clinicians using clinical information, symptoms along with familial information and NGS-based WES tool to decipher the undefined disorders that mimic a known phenotype.

With NGS platforms' availability, personalized medication got more confidence for the individual with unclear or rare diseases. Among different sequencing approaches, WES has always 
been considered a cost-effective approach in molecular diagnostics of rare disorders, it covers all the functional and defined segments (UTR regions, Promoter, exonic) of the genome thus makes it easier to understand, although chances of getting defined drug target genes are moderate so, specified drug or therapy can be used for the treatment. The use of NGS in precision medicine is quite common in complex disorders such as cancers. Several studies on cancer patients exemplify the role of NGS in precision medicine, few of them have been discussed herewith.

NGS has now been increasingly used in the discovery of therapeutic biomarkers of cancers. Beltran et al. applied WES to 97 patients and observed 16 somatic mutations per individual. Based on the findings, recommendations were made to access clinical trials and/or off-labeled use of drugs [76]. A combined study of WES and RNA sequencing (RNAseq) provided the somatic and germline sequencing were highly concordant and yield novel biomarkers for precision medicine [77]. Similarly, WES detected $31 \%$ of the disease-associated somatic mutations in a group of 165 patients, whereas the remaining somatic mutations had preclinical or inferential evidence. On the other hand, 806 germ-line variants were detected out of which 5\% were found to behaving clinical significance and the remaining 56\% with unfamiliar relevance. The study provided insights into the implementation of clinical sequencing programs in cancer precision medicine [78].

WES provides insights into monogenic diseases by searching novel biomarkers for possible therapeutic decisions. A male child (aged 15 months) had Crohn's disease-like illness, encountered via WES diagnosis and 16,124 variations were detected. With subsequent analysis, a novel missense hemizygous mutation was detected in the X-linked inhibitor gene of apoptosis. The mutation was not previously associated with Crohn's disease. Moreover, the mutation was found to have an essential role in the pro-inflammatory response. The mutation was later confirmed by Sanger sequencing and functional aspects were studied using functional assays. Based on all the evidence gathered from the clinical, genetic and functional records, the child was diagnosed by X- linked inhibitor of apoptosis deficiency. Allogeneic hematopoietic progenitor cell transplant was done to avert the development of hemophagocytic lymphohistiocytosis (HLH) [79].

Similarly, in another study, a 14-year-old girl who suffered from autoimmune enteropathy (AIE) was diagnosed with a de novo mutation in the CTLA4 gene using WES. Abatacept (an approved drug), a fusion protein of the CTLA4 extracellular domain was administered that ultimately helped in restoring $\mathrm{T}_{\text {reg }}$ cells functions [80].

WES empowers the precision medicine approach for nephrotic and kidney-related disorders. A study using WES not only uncovered the known kidney disease etiology but also yielded a molecular diagnosis for the individuals with end-stage renal disease (ESRD) of unknown etiology [81]. Similarly, in a pediatric cohort, WES powerfully explicates the monogenic and polygenic 
forms of Steroid Resistant Nephrotic Syndrome (SRNS), which led to identifying novel drug targets for evidence-based clinical management [82]. These findings support the diagnostic utility of WES across different groups with clinical and genetic heterogeneity of kidney disease and explicit the relevant clinical trials and targeted therapies. In addition to this, WES also evaluates the variants causing a potential risk for transplant recipients.

Figure 2 demonstrates the implication of NGS-WES on targeted drug therapy by uncovering the so-called undiagnosed declared disorders by traditional approaches. Reports of finding novel genetic variants are common during the diagnosis of rare disorders, which aids in developing new drug targets. The only bottleneck of personalized medication is there in case of rare diseases that may be limited to a specific cohort of patients. With the subsequent new sequencing technologies, precision medicine reinforces effective treatment of orphan disorders, which was earlier considered as science fiction in the field of medical genetics.

\section{CONCLUSION}

With the advent of NGS, diagnosis of the genetic disorder has become effortless and costeffective. Conventional methods such as; FISH, CGH, karyotyping, etc., have limitations after a certain point. They can help in identifying known genes and their variants associated with the disease but makes them difficult to distinguish the undiagnosed, heterogeneous and rare disorders. Whereas, WES exhibit a clear advantage in identifying the novel genetic cause for several undiagnosed heterogeneous diseases. Recently, WES has come up as a convenient tool for the diagnosis of prenatal and perinatal diagnosis.

Exomes are the functional or protein-coding region of the genome that constitutes about $1 \%$ of the whole genome, but interestingly it harbors $85 \%$ of the mutations contributing to several monogenic conditions. WES has come up to be a handy and essential tool in diagnosing previously categorized undiagnosed disorders by traditional technologies. Exome sequencing targets that $1 \%$ of the functional part of the genome that ultimately reduces the cost of the diagnosis. With the arrival of WES, the diagnostic yield has increased by $25 \%$ for the previously undiagnosed genetic disorders.

Multiple WES studies have identified the novel variations in pediatric-onset genetic diseases, but those unique genomic variants require our attention to set up pharmacogenomic blueprints for personalized and precision medicine. WES followed by in-silico detection of novel functionally relevant variants can be made pharmacogenomic targets for drug discovery. Finally, we recommend 
the much reliable and cost-effective WES strategy to be used as the first-line diagnostic tool for premarital and prenatal genetic testing to prevent the heritable risk in future generations.

\section{ACKNOWLEDGEMENTS}

We thank the Department of Science \& Technology (DST), New Delhi for the financial assistance (File No. DST/INSPIRE/04/2017/001350).

\section{AUTHOR CONTRIBUTIONS}

M.F. drafted sections of the article. M.F., V.S., H.S., wrote the manuscript. M.F. and V.S. revised it critically, contributing crucial intellectual content to the final manuscript. All authors provided final approval of the version to be published.

\section{FUNDING SUPPORT STATEMENT}

The authors have no support or funding to report.

\section{DISCLOSURE STATEMENT}

The authors have declared that no competing interests exist. 


\section{References}

1. Groisman, B.; Bermejo-Sánchez, E.; Romitti, P.A.; Botto, L.D.; Feldkamp, M.L.; Walani, S.R.; Mastroiacovo, P. Join World Birth Defects Day. Pediatric research 2019, 86, 3-4, doi:10.1038/s41390-019-0392-x.

2. McNamara, K.; O'Donoghue, K.; O'Connell, O.; Greene, R.A. Antenatal and Intrapartum Care of Pregnancy Complicated by Lethal Fetal Anomaly. The Obstetrician \& Gynaecologist 2013, 15, 189194, doi:10.1111/tog.12028.

3. Kasparian, N.A.; Kan, J.M.; Sood, E.; Wray, J.; Pincus, H.A.; Newburger, J.W. Mental Health Care for Parents of Babies with Congenital Heart Disease during Intensive Care Unit Admission: Systematic Review and Statement of Best Practice. Early human development 2019, 139, 104837, doi:10.1016/j.earlhumdev.2019.104837.

4. Yang, D.D.; Baujat, G.; Neuraz, A.; Garcelon, N.; Messiaen, C.; Sandrin, A.; Cheron, G.; Burgun, A.; Pejin, Z.; Cormier-Daire, V.; et al. Healthcare Trajectory of Children with Rare Bone Disease Attending Pediatric Emergency Departments. Orphanet journal of rare diseases 2020, 15, 2 , doi:10.1186/s13023-019-1284-1.

5. Pilania, R.K.; Chaudhary, H.; Jindal, A.K.; Rawat, A.; Singh, S. Current Status and Prospects of Primary Immunodeficiency Diseases in Asia. Genes \& Diseases 2020, 7, 3-11, doi:10.1016/j.gendis.2019.09.004.

6. Ontario Health (Quality) Genome-Wide Sequencing for Unexplained Developmental Disabilities or Multiple Congenital Anomalies: A Health Technology Assessment. Ontario Health Technology Assessment Series 2020, 20, 1-178.

7. di Resta, C.; Ferrari, M. Next Generation Sequencing: From Research Area to Clinical Practice. EJIFCC 2018, 29, 215-220.

8. Tan, T.Y.; Dillon, O.J.; Stark, Z.; Schofield, D.; Alam, K.; Shrestha, R.; Chong, B.; Phelan, D.; Brett, G.R.; Creed, E.; et al. Diagnostic Impact and Cost-Effectiveness of Whole-Exome Sequencing for Ambulant Children With Suspected Monogenic Conditions. JAMA pediatrics 2017, 171, 855-862, doi:10.1001/jamapediatrics.2017.1755.

9. Bertier, G.; Hétu, M.; Joly, Y. Unsolved Challenges of Clinical Whole-Exome Sequencing: A Systematic Literature Review of End-Users' Views. BMC medical genomics 2016, 9, 52, doi:10.1186/s12920-016-0213-6.

10. Kaname, T.; Yanagi, K.; Naritomi, K. A Commentary on the Promise of Whole-Exome Sequencing in Medical Genetics. Journal of human genetics 2014, 59, 117-8, doi:10.1038/jhg.2014.7.

11. Ng, S.B.; Buckingham, K.J.; Lee, C.; Bigham, A.W.; Tabor, H.K.; Dent, K.M.; Huff, C.D.; Shannon, P.T.; Jabs, E.W.; Nickerson, D.A.; et al. Exome Sequencing Identifies the Cause of a Mendelian Disorder. Nature genetics 2010, 42, 30-5, doi:10.1038/ng.499.

12. Bertier, G.; Sénécal, K.; Borry, P.; Vears, D.F. Unsolved Challenges in Pediatric Whole-Exome Sequencing: A Literature Analysis. Critical reviews in clinical laboratory sciences 2017, 54, 134-142, doi:10.1080/10408363.2016.1275516.

13. Best, S.; Wou, K.; Vora, N.; van der Veyver, I.B.; Wapner, R.; Chitty, L.S. Promises, Pitfalls and Practicalities of Prenatal Whole Exome Sequencing. Prenatal diagnosis 2018, 38, 10-19, doi:10.1002/pd.5102. 
14. Iossifov, I.; O’Roak, B.J.; Sanders, S.J.; Ronemus, M.; Krumm, N.; Levy, D.; Stessman, H.A.; Witherspoon, K.T.; Vives, L.; Patterson, K.E.; et al. The Contribution of de Novo Coding Mutations to Autism Spectrum Disorder. Nature 2014, 515, 216-21, doi:10.1038/nature13908.

15. Jamuar, S.S.; Lam, A.-T.N.; Kircher, M.; D'Gama, A.M.; Wang, J.; Barry, B.J.; Zhang, X.; Hill, R.S.; Partlow, J.N.; Rozzo, A.; et al. Somatic Mutations in Cerebral Cortical Malformations. The New England journal of medicine 2014, 371, 733-43, doi:10.1056/NEJMoa1314432.

16. Epi4K Consortium; Epilepsy Phenome/Genome Project; Allen, A.S.; Berkovic, S.F.; Cossette, P.; Delanty, N.; Dlugos, D.; Eichler, E.E.; Epstein, M.P.; Glauser, T.; et al. De Novo Mutations in Epileptic Encephalopathies. Nature 2013, 501, 217-21, doi:10.1038/nature12439.

17. Zaidi, S.; Choi, M.; Wakimoto, H.; Ma, L.; Jiang, J.; Overton, J.D.; Romano-Adesman, A.; Bjornson, R.D.; Breitbart, R.E.; Brown, K.K.; et al. De Novo Mutations in Histone-Modifying Genes in Congenital Heart Disease. Nature 2013, 498, 220-3, doi:10.1038/nature12141.

18. Deciphering Developmental Disorders Study Large-Scale Discovery of Novel Genetic Causes of Developmental Disorders. Nature 2015, 519, 223-8, doi:10.1038/nature14135.

19. Lemay, P.; de Marco, P.; Traverso, M.; Merello, E.; Dionne-Laporte, A.; Spiegelman, D.; Henrion, É.; Diallo, O.; Audibert, F.; Michaud, J.L.; et al. Whole Exome Sequencing Identifies Novel Predisposing Genes in Neural Tube Defects. Molecular genetics \& genomic medicine 2019, 7, e00467, doi:10.1002/mgg3.467.

20. Petrovski, S.; Aggarwal, V.; Giordano, J.L.; Stosic, M.; Wou, K.; Bier, L.; Spiegel, E.; Brennan, K.; Stong, N.; Jobanputra, V.; et al. Whole-Exome Sequencing in the Evaluation of Fetal Structural Anomalies: A Prospective Cohort Study. Lancet (London, England) 2019, 393, 758-767, doi:10.1016/S0140-6736(18)32042-7.

21. Fareed, M.; Afzal, M. Genetics of Consanguinity and Inbreeding in Health and Disease. Annals of human biology 2017, 44, 99-107, doi:10.1080/03014460.2016.1265148.

22. Fareed, Mo.; Afzal, M. Evidence of Inbreeding Depression on Height, Weight, and Body Mass Index: A Population-Based Child Cohort Study. American Journal of Human Biology 2014, 26, 784795, doi:10.1002/ajhb.22599.

23. Fareed, M.; Afzal, M. Estimating the Inbreeding Depression on Cognitive Behavior: A Population Based Study of Child Cohort. PloS one 2014, 9, e109585, doi:10.1371/journal.pone.0109585.

24. Fareed, M.; Kaisar Ahmad, M.; Azeem Anwar, M.; Afzal, M. Impact of Consanguineous Marriages and Degrees of Inbreeding on Fertility, Child Mortality, Secondary Sex Ratio, Selection Intensity, and Genetic Load: A Cross-Sectional Study from Northern India. Pediatric research 2017, 81, 18 26, doi:10.1038/pr.2016.177.

25. Altmann, H.M.; Tester, D.J.; Will, M.L.; Middha, S.; Evans, J.M.; Eckloff, B.W.; Ackerman, M.J. Homozygous/Compound Heterozygous Triadin Mutations Associated With Autosomal-Recessive Long-QT Syndrome and Pediatric Sudden Cardiac Arrest: Elucidation of the Triadin Knockout Syndrome. Circulation 2015, 131, 2051-60, doi:10.1161/CIRCULATIONAHA.115.015397.

26. Liew, W.K.M.; Ben-Omran, T.; Darras, B.T.; Prabhu, S.P.; de Vivo, D.C.; Vatta, M.; Yang, Y.; Eng, C.M.; Chung, W.K. Clinical Application of Whole-Exome Sequencing: A Novel Autosomal Recessive Spastic Ataxia of Charlevoix-Saguenay Sequence Variation in a Child with Ataxia. JAMA neurology 2013, 70, 788-91, doi:10.1001/jamaneurol.2013.247. 
27. Srikajon, J.; Pitakpatapee, Y.; Limwongse, C.; Chirapapaisan, N.; Srivanitchapoom, P. Autosomal Recessive Spastic Ataxia of Charlevoix-Saguenay (ARSACS) in a Thai Patient: The Classic Clinical Manifestations, Funduscopic Feature, and Brain Imaging Findings with a Novel Mutation in the SACS Gene. Tremor and other hyperkinetic movements (New York, N.Y.) 2020, 10, 1, doi:10.5334/tohm.68.

28. Sawyer, S.L.; Schwartzentruber, J.; Beaulieu, C.L.; Dyment, D.; Smith, A.; Warman Chardon, J.; Yoon, G.; Rouleau, G.A.; Suchowersky, O.; Siu, V.; et al. Exome Sequencing as a Diagnostic Tool for Pediatric-Onset Ataxia. Human mutation 2014, 35, 45-9, doi:10.1002/humu.22451.

29. Makrythanasis, P.; Nelis, M.; Santoni, F.A.; Guipponi, M.; Vannier, A.; Béna, F.; Gimelli, S.; Stathaki, E.; Temtamy, S.; Mégarbané, A.; et al. Diagnostic Exome Sequencing to Elucidate the Genetic Basis of Likely Recessive Disorders in Consanguineous Families. Human mutation 2014, 35, 1203-10, doi:10.1002/humu.22617.

30. Shamriz, O.; Shaag, A.; Yaacov, B.; NaserEddin, A.; Weintraub, M.; Elpeleg, O.; Stepensky, P. The Use of Whole Exome Sequencing for the Diagnosis of Autosomal Recessive Malignant Infantile Osteopetrosis. Clinical genetics 2017, 92, 80-85, doi:10.1111/cge.12804.

31. Hao, X.; Liu, S.; Dong, Q.; Zhang, H.; Zhao, J.; Su, L. Whole Exome Sequencing Identifies Recessive PKHD1 Mutations in a Chinese Twin Family with Caroli Disease. PloS one 2014, 9, e92661, doi:10.1371/journal.pone.0092661.

32. Germain, D.P. General Aspects of X-Linked Diseases; 2006; ISBN 190353903X.

33. Al-Mubarak, B.; Abouelhoda, M.; Omar, A.; AlDhalaan, H.; Aldosari, M.; Nester, M.; Alshamrani, H.A.; El-Kalioby, M.; Goljan, E.; Albar, R.; et al. Whole Exome Sequencing Reveals Inherited and de Novo Variants in Autism Spectrum Disorder: A Trio Study from Saudi Families. Scientific reports 2017, 7, 5679, doi:10.1038/s41598-017-06033-1.

34. Bruun, T.U.J.; DesRoches, C.-L.; Wilson, D.; Chau, V.; Nakagawa, T.; Yamasaki, M.; Hasegawa, S.; Fukao, T.; Marshall, C.; Mercimek-Andrews, S. Prospective Cohort Study for Identification of Underlying Genetic Causes in Neonatal Encephalopathy Using Whole-Exome Sequencing. Genetics in medicine : official journal of the American College of Medical Genetics 2018, 20, 486-494, doi:10.1038/gim.2017.129.

35. Srivastava, S.; Cohen, J.S.; Vernon, H.; Barañano, K.; McClellan, R.; Jamal, L.; Naidu, S.; Fatemi, A. Clinical Whole Exome Sequencing in Child Neurology Practice. Annals of neurology 2014, 76, 47383, doi:10.1002/ana.24251.

36. Yang, Y.; Muzny, D.M.; Xia, F.; Niu, Z.; Person, R.; Ding, Y.; Ward, P.; Braxton, A.; Wang, M.; Buhay, C.; et al. Molecular Findings among Patients Referred for Clinical Whole-Exome Sequencing. JAMA 2014, 312, 1870-9, doi:10.1001/jama.2014.14601.

37. Tong, W.; Wang, Y.; Lu, Y.; Ye, T.; Song, C.; Xu, Y.; Li, M.; Ding, J.; Duan, Y.; Zhang, L.; et al. WholeExome Sequencing Helps the Diagnosis and Treatment in Children with Neurodevelopmental Delay Accompanied Unexplained Dyspnea. Scientific reports 2018, 8, 5214, doi:10.1038/s41598-01823503-2.

38. Thorburn, D.R. Mitochondrial Disorders: Prevalence, Myths and Advances. Journal of inherited metabolic disease 2004, 27, 349-62, doi:10.1023/B:BOLI.0000031098.41409.55. 
39. Luft, R. The Development of Mitochondrial Medicine. Proceedings of the National Academy of Sciences of the United States of America 1994, 91, 8731-8, doi:10.1073/pnas.91.19.8731.

40. Chinnery, P.F. Mitochondrial Disorders Overview; 1993;

41. Wolf, N.I.; Smeitink, J.A.M. Mitochondrial Disorders: A Proposal for Consensus Diagnostic Criteria in Infants and Children. Neurology 2002, 59, 1402-5, doi:10.1212/01.wnl.0000031795.91814.d8.

42. Enns, G.M. Pediatric Mitochondrial Diseases and the Heart. Current opinion in pediatrics 2017, 29, 541-551, doi:10.1097/MOP.0000000000000535.

43. Walker, U.A.; Collins, S.; Byrne, E. Respiratory Chain Encephalomyopathies: A Diagnostic Classification. European neurology 1996, 36, 260-7, doi:10.1159/000117269.

44. Koenig, M.K. Presentation and Diagnosis of Mitochondrial Disorders in Children. Pediatric neurology 2008, 38, 305-13, doi:10.1016/j.pediatrneurol.2007.12.001.

45. Seo, G.H.; Oh, A.; Kim, E.N.; Lee, Y.; Park, J.; Kim, T.; Lim, Y.-M.; Kim, G.-H.; Kim, C.J.; Yoo, H.-W.; et al. Identification of Extremely Rare Mitochondrial Disorders by Whole Exome Sequencing. Journal of human genetics 2019, 64, 1117-1125, doi:10.1038/s10038-019-0660-y.

46. Thompson, K.; Collier, J.J.; Glasgow, R.I.C.; Robertson, F.M.; Pyle, A.; Blakely, E.L.; Alston, C.L.; Oláhová, M.; McFarland, R.; Taylor, R.W. Recent Advances in Understanding the Molecular Genetic Basis of Mitochondrial Disease. Journal of inherited metabolic disease 2020, 43, 36-50, doi:10.1002/jimd.12104.

47. Pronicka, E.; Piekutowska-Abramczuk, D.; Ciara, E.; Trubicka, J.; Rokicki, D.; KarkucińskaWięckowska, A.; Pajdowska, M.; Jurkiewicz, E.; Halat, P.; Kosińska, J.; et al. New Perspective in Diagnostics of Mitochondrial Disorders: Two Years' Experience with Whole-Exome Sequencing at a National Paediatric Centre. Journal of translational medicine 2016, 14, 174, doi:10.1186/s12967016-0930-9.

48. Götz, A.; Tyynismaa, H.; Euro, L.; Ellonen, P.; Hyötyläinen, T.; Ojala, T.; Hämäläinen, R.H.; Tommiska, J.; Raivio, T.; Oresic, M.; et al. Exome Sequencing Identifies Mitochondrial Alanyl-TRNA Synthetase Mutations in Infantile Mitochondrial Cardiomyopathy. American journal of human genetics 2011, 88, 635-42, doi:10.1016/j.ajhg.2011.04.006.

49. Taylor, R.W.; Pyle, A.; Griffin, H.; Blakely, E.L.; Duff, J.; He, L.; Smertenko, T.; Alston, C.L.; Neeve, V.C.; Best, A.; et al. Use of Whole-Exome Sequencing to Determine the Genetic Basis of Multiple Mitochondrial Respiratory Chain Complex Deficiencies. JAMA 2014, 312, 68-77, doi:10.1001/jama.2014.7184.

50. Walani, S.R.; Biermann, J. March of Dimes Foundation: Leading the Way to Birth Defects Prevention. Public health reviews 38, 12, doi:10.1186/s40985-017-0058-3.

51. Shannon, G.D.; Alberg, C.; Nacul, L.; Pashayan, N. Preconception Healthcare and Congenital Disorders: Systematic Review of the Effectiveness of Preconception Care Programs in the Prevention of Congenital Disorders. Maternal and child health journal 2014, 18, 1354-79, doi:10.1007/s10995-013-1370-2.

52. Grunewald, S.; Matthijs, G.; Jaeken, J. Congenital Disorders of Glycosylation: A Review. Pediatric research 2002, 52, 618-24, doi:10.1203/00006450-200211000-00003. 
53. Timal, S.; Hoischen, A.; Lehle, L.; Adamowicz, M.; Huijben, K.; Sykut-Cegielska, J.; Paprocka, J.; Jamroz, E.; van Spronsen, F.J.; Körner, C.; et al. Gene Identification in the Congenital Disorders of Glycosylation Type I by Whole-Exome Sequencing. Human molecular genetics 2012, 21, 4151-61, doi:10.1093/hmg/dds123.

54. Zhang, Z.; Huang, T.-L.; Ma, J.; He, W.-J.; Gu, H. Clinical and Whole-Exome Sequencing Findings in Two Siblings from Hani Ethnic Minority with Congenital Glycosylation Disorders. BMC medical genetics 2019, 20, 181, doi:10.1186/s12881-019-0902-z.

55. Nambot, S.; Thevenon, J.; Kuentz, P.; Duffourd, Y.; Tisserant, E.; Bruel, A.-L.; Mosca-Boidron, A.-L.; Masurel-Paulet, A.; Lehalle, D.; Jean-Marçais, N.; et al. Clinical Whole-Exome Sequencing for the Diagnosis of Rare Disorders with Congenital Anomalies and/or Intellectual Disability: Substantial Interest of Prospective Annual Reanalysis. Genetics in medicine : official journal of the American College of Medical Genetics 2018, 20, 645-654, doi:10.1038/gim.2017.162.

56. Bekheirnia, M.R.; Bekheirnia, N.; Bainbridge, M.N.; Gu, S.; Coban Akdemir, Z.H.; Gambin, T.; Janzen, N.K.; Jhangiani, S.N.; Muzny, D.M.; Michael, M.; et al. Whole-Exome Sequencing in the Molecular Diagnosis of Individuals with Congenital Anomalies of the Kidney and Urinary Tract and Identification of a New Causative Gene. Genetics in medicine : official journal of the American College of Medical Genetics 2017, 19, 412-420, doi:10.1038/gim.2016.131.

57. Johnson, S.R.; Leo, P.J.; Mclnerney-Leo, A.M.; Anderson, L.K.; Marshall, M.; McGown, I.; Newell, F.; Brown, M.A.; Conwell, L.S.; Harris, M.; et al. Whole-Exome Sequencing for Mutation Detection in Pediatric Disorders of Insulin Secretion: Maturity Onset Diabetes of the Young and Congenital Hyperinsulinism. Pediatric diabetes 2018, 19, 656-662, doi:10.1111/pedi.12638.

58. Simonato, M.; Bennett, J.; Boulis, N.M.; Castro, M.G.; Fink, D.J.; Goins, W.F.; Gray, S.J.; Lowenstein, P.R.; Vandenberghe, L.H.; Wilson, T.J.; et al. Progress in Gene Therapy for Neurological Disorders. Nature reviews. Neurology 2013, 9, 277-91, doi:10.1038/nrneurol.2013.56.

59. Epi4K Consortium; Epilepsy Phenome/Genome Project; Allen, A.S.; Berkovic, S.F.; Cossette, P.; Delanty, N.; Dlugos, D.; Eichler, E.E.; Epstein, M.P.; Glauser, T.; et al. De Novo Mutations in Epileptic Encephalopathies. Nature 2013, 501, 217-21, doi:10.1038/nature12439.

60. Rosewich, H.; Thiele, H.; Ohlenbusch, A.; Maschke, U.; Altmüller, J.; Frommolt, P.; Zirn, B.; Ebinger, F.; Siemes, H.; Nürnberg, P.; et al. Heterozygous De-Novo Mutations in ATP1A3 in Patients with Alternating Hemiplegia of Childhood: A Whole-Exome Sequencing Gene-Identification Study. The Lancet. Neurology 2012, 11, 764-73, doi:10.1016/S1474-4422(12)70182-5.

61. Worthey, E.A.; Raca, G.; Laffin, J.J.; Wilk, B.M.; Harris, J.M.; Jakielski, K.J.; Dimmock, D.P.; Strand, E.A.; Shriberg, L.D. Whole-Exome Sequencing Supports Genetic Heterogeneity in Childhood Apraxia of Speech. Journal of neurodevelopmental disorders 2013, 5, 29, doi:10.1186/1866-19555-29.

62. Dyment, D.A.; Tétreault, M.; Beaulieu, C.L.; Hartley, T.; Ferreira, P.; Chardon, J.W.; Marcadier, J.; Sawyer, S.L.; Mosca, S.J.; Innes, A.M.; et al. Whole-Exome Sequencing Broadens the Phenotypic Spectrum of Rare Pediatric Epilepsy: A Retrospective Study. Clinical genetics 2015, 88, 34-40, doi:10.1111/cge.12464. 
63. Fareed, M.; Afzal, M. Single Nucleotide Polymorphism in Genome-Wide Association of Human Population: A Tool for Broad Spectrum Service. Egyptian Journal of Medical Human Genetics 2013, 14, 123-134, doi:10.1016/j.ejmhg.2012.08.001.

64. McClellan, J.; King, M.-C. Genetic Heterogeneity in Human Disease. Cell 2010, 141, 210-7, doi:10.1016/j.cell.2010.03.032.

65. Bouchoucha, S.; Chikhaoui, A.; Najjar, D.; Dallali, H.; Khammessi, M.; Abdelhak, S.; Nessibe, N.; Shboul, M.; Kircher, S.G.; al Kaissi, A.; et al. Clinical and Genetic Heterogeneity in Six Tunisian Families With Horizontal Gaze Palsy With Progressive Scoliosis: A Retrospective Study of 13 Cases. Frontiers in pediatrics 2020, 8, 172, doi:10.3389/fped.2020.00172.

66. McMichael, G.; Bainbridge, M.N.; Haan, E.; Corbett, M.; Gardner, A.; Thompson, S.; van Bon, B.W.M.; van Eyk, C.L.; Broadbent, J.; Reynolds, C.; et al. Whole-Exome Sequencing Points to Considerable Genetic Heterogeneity of Cerebral Palsy. Molecular psychiatry 2015, 20, 176-82, doi:10.1038/mp.2014.189.

67. Lee, J.S.; Yoo, T.; Lee, M.; Lee, Y.; Jeon, E.; Kim, S.Y.; Lim, B.C.; Kim, K.J.; Choi, M.; Chae, J.-H. Genetic Heterogeneity in Leigh Syndrome: Highlighting Treatable and Novel Genetic Causes. Clinical genetics 2020, 97, 586-594, doi:10.1111/cge.13713.

68. Arai-Ichinoi, N.; Uematsu, M.; Sato, R.; Suzuki, T.; Kudo, H.; Kikuchi, A.; Hino-Fukuyo, N.; Matsumoto, M.; Igarashi, K.; Haginoya, K.; et al. Genetic Heterogeneity in 26 Infants with a Hypomyelinating Leukodystrophy. Human genetics 2016, 135, 89-98, doi:10.1007/s00439-0151617-7.

69. Zhu, X.; Petrovski, S.; Xie, P.; Ruzzo, E.K.; Lu, Y.-F.; McSweeney, K.M.; Ben-Zeev, B.; Nissenkorn, A.; Anikster, Y.; Oz-Levi, D.; et al. Whole-Exome Sequencing in Undiagnosed Genetic Diseases: Interpreting 119 Trios. Genetics in medicine : official journal of the American College of Medical Genetics 2015, 17, 774-81, doi:10.1038/gim.2014.191.

70. Mahler, E.A.; Johannsen, J.; Tsiakas, K.; Kloth, K.; Lüttgen, S.; Mühlhausen, C.; Alhaddad, B.; Haack, T.B.; Strom, T.M.; Kortüm, F.; et al. Exome Sequencing in Children. Deutsches Arzteblatt international 2019, 116, 197-204, doi:10.3238/arztebl.2019.0197.

71. Gahl, W.A.; Markello, T.C.; Toro, C.; Fajardo, K.F.; Sincan, M.; Gill, F.; Carlson-Donohoe, H.; Gropman, A.; Pierson, T.M.; Golas, G.; et al. The National Institutes of Health Undiagnosed Diseases Program: Insights into Rare Diseases. Genetics in medicine : official journal of the American College of Medical Genetics 2012, 14, 51-9, doi:10.1038/gim.0b013e318232a005.

72. Kuperberg, M.; Lev, D.; Blumkin, L.; Zerem, A.; Ginsberg, M.; Linder, I.; Carmi, N.; Kivity, S.; Lerman-Sagie, T.; Leshinsky-Silver, E. Utility of Whole Exome Sequencing for Genetic Diagnosis of Previously Undiagnosed Pediatric Neurology Patients. Journal of child neurology 2016, 31, 15341539, doi:10.1177/0883073816664836.

73. Duan, C.; Wang, H.; Chen, Y.; Chu, P.; Xing, T.; Gao, C.; Yue, Z.; Zheng, J.; Jin, M.; Gu, W.; et al. Whole Exome Sequencing Reveals Novel Somatic Alterations in Neuroblastoma Patients with Chemotherapy. Cancer Cell International 2018, 18, doi:10.1186/s12935-018-0521-3.

74. D'Gama, A.M.; Walsh, C.A. Somatic Mosaicism and Neurodevelopmental Disease. Nature Neuroscience 2018, 21, 1504-1514, doi:10.1038/s41593-018-0257-3. 
75. Rennert, H.; Eng, K.; Zhang, T.; Tan, A.; Xiang, J.; Romanel, A.; Kim, R.; Tam, W.; Liu, Y.C.; Bhinder, B.; et al. Development and Validation of a Whole-Exome Sequencing Test for Simultaneous Detection of Point Mutations, Indels and Copy-Number Alterations for Precision Cancer Care. npj Genomic Medicine 2016, 1, doi:10.1038/npjgenmed.2016.19.

76. Beltran, H.; Eng, K.; Mosquera, J.M.; Sigaras, A.; Romanel, A.; Rennert, H.; Kossai, M.; Pauli, C.; Faltas, B.; Fontugne, J.; et al. Whole-Exome Sequencing of Metastatic Cancer and Biomarkers of Treatment Response. JAMA oncology 2015, 1, 466-74, doi:10.1001/jamaoncol.2015.1313.

77. van Allen, E.M.; Robinson, D.; Morrissey, C.; Pritchard, C.; Imamovic, A.; Carter, S.; Rosenberg, M.; McKenna, A.; Wu, Y.-M.; Cao, X.; et al. A Comparative Assessment of Clinical Whole Exome and Transcriptome Profiling across Sequencing Centers: Implications for Precision Cancer Medicine. Oncotarget 2016, 7, 52888-52899, doi:10.18632/oncotarget.9184.

78. Ghazani, A.A.; Oliver, N.M.; St Pierre, J.P.; Garofalo, A.; Rainville, I.R.; Hiller, E.; Treacy, D.J.; RojasRudilla, V.; Wood, S.; Bair, E.; et al. Assigning Clinical Meaning to Somatic and Germ-Line WholeExome Sequencing Data in a Prospective Cancer Precision Medicine Study. Genetics in medicine : official journal of the American College of Medical Genetics 2017, 19, 787-795, doi:10.1038/gim.2016.191.

79. Worthey, E.A.; Mayer, A.N.; Syverson, G.D.; Helbling, D.; Bonacci, B.B.; Decker, B.; Serpe, J.M.; Dasu, T.; Tschannen, M.R.; Veith, R.L.; et al. Making a Definitive Diagnosis: Successful Clinical Application of Whole Exome Sequencing in a Child with Intractable Inflammatory Bowel Disease. Genetics in medicine : official journal of the American College of Medical Genetics 2011, 13, 25562, doi:10.1097/GIM.0b013e3182088158.

80. Lee, S.; Moon, J.S.; Lee, C.-R.; Kim, H.-E.; Baek, S.-M.; Hwang, S.; Kang, G.H.; Seo, J.K.; Shin, C.H.; Kang, H.J.; et al. Abatacept Alleviates Severe Autoimmune Symptoms in a Patient Carrying a de Novo Variant in CTLA-4. The Journal of allergy and clinical immunology 2016, 137, 327-330, doi:10.1016/j.jaci.2015.08.036.

81. Mann, N.; Braun, D.A.; Amann, K.; Tan, W.; Shril, S.; Connaughton, D.M.; Nakayama, M.; Schneider, R.; Kitzler, T.M.; van der Ven, A.T.; et al. Whole-Exome Sequencing Enables a Precision Medicine Approach for Kidney Transplant Recipients. Journal of the American Society of Nephrology : JASN 2019, 30, 201-215, doi:10.1681/ASN.2018060575.

82. Bierzynska, A.; McCarthy, H.J.; Soderquest, K.; Sen, E.S.; Colby, E.; Ding, W.Y.; Nabhan, M.M.; Kerecuk, L.; Hegde, S.; Hughes, D.; et al. Genomic and Clinical Profiling of a National Nephrotic Syndrome Cohort Advocates a Precision Medicine Approach to Disease Management. Kidney international 2017, 91, 937-947, doi:10.1016/j.kint.2016.10.013. 


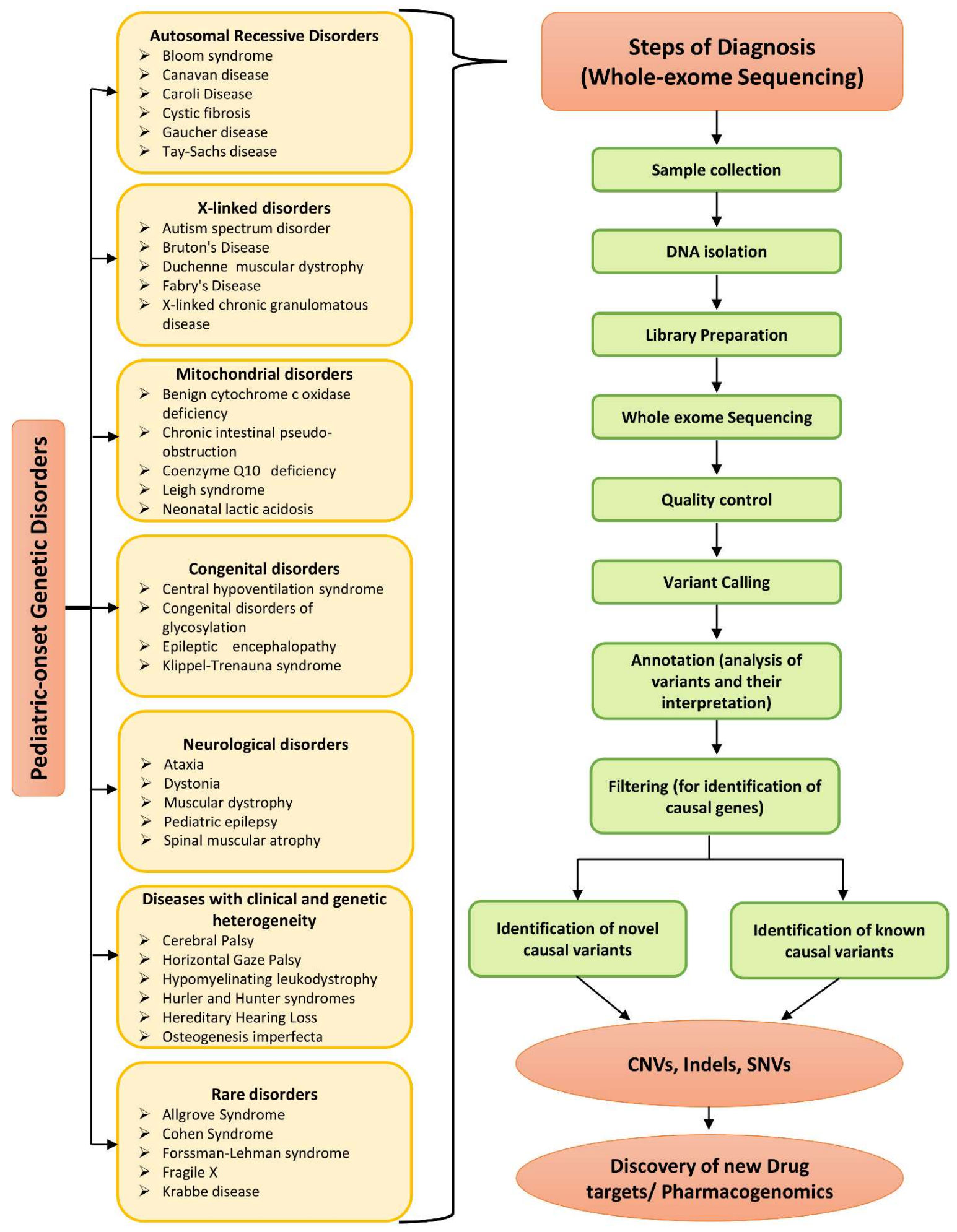

Figure 1. Schematic diagram depicting an overview of pediatric-onset genetic disorders and the complete workflow of diagnosis of genetic disorders using whole-exome sequencing (WES). WES generates disease-associated common and novel variants, which further be used as pharmacogenomic targets for drug discovery. 
(I) Traditional Diagnosis and Medical treatment
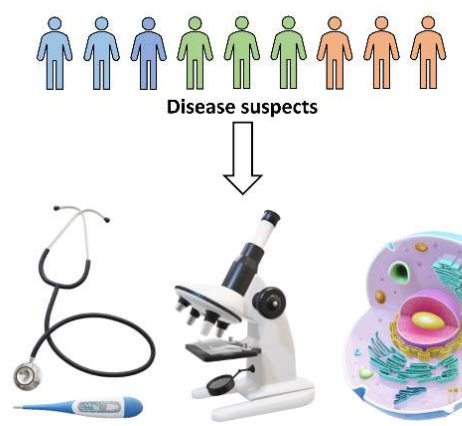

Diagnosis

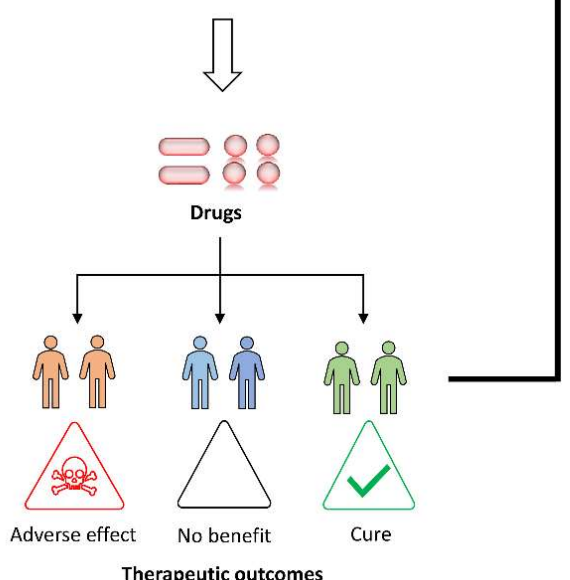

(II) Whole-exome sequencing (NGS-WES) for the diagnosis and treatment of undiagnosed genetic disorders
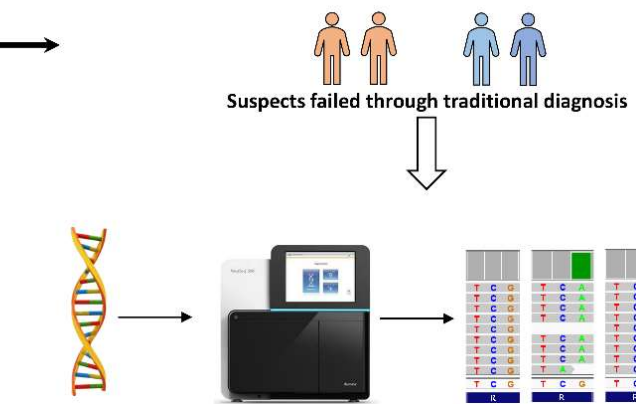

Genomic sample

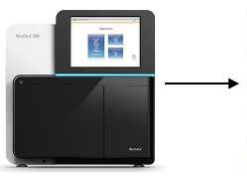

NGS

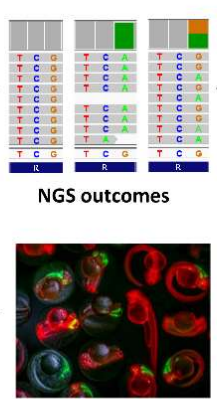

Functional analysis $\rrbracket$ Pharmacogenomics

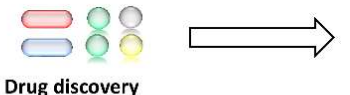

Drug discovery

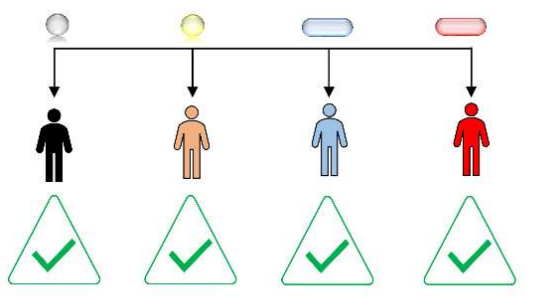

Personalised \& precision medication

Figure 2. Graphical illustration of diagnostic and therapeutic advantages of whole-exome sequencing (WES) over conventional testing and curative methods. Traditional approaches generally fail to distinguish the pleiotrophic, polygenic, compound heterozygous and sometimes even monogenic traits. WES, using the NGS platform, explicitly investigate the confounding disease etiology and uncover the novel drug targets for precision medicine. 